\title{
ETS2 and microRNA-155 regulate the pathogenesis of heart failure through targeting and regulating GPR18 expression
}

\author{
JING LI, HONGLING SU, YAN ZHU, YUNSHAN CAO and XUMING MA \\ Department of Cardiology, Gansu Provincial Hospital, Lanzhou, Gansu 730000, P.R. China
}

Received June 13, 2018; Accepted November 29, 2019

DOI: $10.3892 / \mathrm{etm} .2020 .8642$

\begin{abstract}
Heart failure (HF) is a global pandemic cardiovascular disease with increasing prevalence, but the pathogenesis remains to be elucidated. The present study aimed to investigate the underlying mechanism in heart failure (HF) using bioinformatics and experimental validation. A HF-associated dataset GSE84796 was downloaded from the Gene Expression Omnibus database and differentially expressed genes (DEGs) were screened for using Bayes method in the Limma package. Kyoto Encyclopedia of Genes and Genomes pathway analysis was used to perform pathway enrichment analysis of these DEGs using The Database for Annotation, Visualization and Integrated Discovery. A protein-protein interaction (PPI) network of DEG-encoded proteins was subsequently constructed using the Search tool for the Retrieval of Interacting Genes/Proteins, and a transcription factor (TF)/miRNA-target network was constructed according to the WEB-based Gene SeT AnaLysis Tookit. The expression levels of microRNA (miRNA/miR)-155, G-protein coupled receptor 18 (GRP18) and E26 transformation-specific transcription factor 2 (ETS2) were analyzed in clinical HF samples, and functional validations were performed in H9c2 (2-1) cells. A total of 419 DEGs were identified, including 366 upregulated genes and 53 downregulated genes. The upregulated DEGs were significantly enriched in the pathways of 'cytokine-cytokine receptor interaction', 'natural killer cell mediated cytotoxicity' and 'primary immunodeficiency'. A total of two functional modules were identified in the PPI network: Module A was enriched in 3 KEGG pathways and module B was enriched in 15 KEGG pathways. Furthermore, a total of three miRNAs and eight TFs were identified in the TF/miRNA-target network. Specifically, GPR18 was discovered to be targeted by both ETS2 and miR-155. Clinical validation revealed that the expression levels
\end{abstract}

Correspondence to: Dr Xuming Ma, Department of Cardiology, Gansu Provincial Hospital, 204 Donggangxi Road, Lanzhou, Gansu 730000, P.R. China

E-mail: xumingmazxc@163.com

Key words: heart failure, regulatory network, cell viability, apoptosis, E26 transformation-specific transcription factor 2, G-protein coupled receptor 18 of miR-155 were significantly decreased in the HF samples, whereas the expression levels of ETS2 and GPR18 were significantly increased in HF samples. In conclusion, the present study suggested that GPR18 may be a target of ETS2 and miR-155, and miR-155 may regulate cell viability and apoptosis in H9c2 (2-1) cells through targeting and regulating GPR18.

\section{Introduction}

Heart failure (HF) is a common health problem worldwide, which leads to disability and reductions in life quality and expectancy (1); however, because HF is prevalent in elderly populations, the management of HF is often associated with a high use of resources and healthcare costs (2). For example, in China it is estimated that HF healthcare has cost $\sim \$ 5.42$ billion per year, accounting for $5.01 \%$ of total healthcare costs (3). Although a number of different methods have been investigated to help manage $\mathrm{HF}$, including medical devices, pharmacological agents and telemonitoring, the clinical treatment of HF remains relatively poor (4). Therefore, there is an urgent requirement for clinicians to investigate novel therapies for the management of HF.

The dysregulation of gene expression is a common mechanism that occurs in HF and microRNAs (miRNAs) are suggested to serve critical roles in the metabolic modulation during HF (5). Within the past few decades, concerted efforts have been made to investigate the mechanism of HF. For example, previous studies have revealed that miRNA (miR)-214 is upregulated in HF and suppresses the transcription factor (TF) X-box binding protein 1 (XBP1)-mediated endothelial cell angiogenesis (6,7). Masson et al (8) reported that increased levels of circulating miR-132 improved the hospitalization of patients with HF, whilst miR-21 was observed to negatively regulate $\mathrm{T}$ regulatory cells in coronary heart disease via the transforming growth factor- $\beta 1 /$ Smad pathway, including HF (9). Moreover, TFs are considered to serve important roles in the regulation of gene expression (10); Bakker et al (11) demonstrated that T-box TF 3 (TBX3) could reprogram mature cardiac myocytes into pacemaker-like cells, whereas the silenced TF interferon regulatory factor 5 (IRF5) was indicated to reprogram the macrophage phenotype and promote infarct healing (12). In addition, cardiomyocyte-specific IkB kinase $(\mathrm{IKK}) / \mathrm{NF}-\kappa \mathrm{B}$ activation reversed inflammation in HF (13). However, despite these findings, the pathogenesis of HF is still not fully understood. 
To identify the mechanisms of HF, Chevillard et al have created a dataset (accession no. GSE84796) on the Gene Expression Omnibus (GEO) database to investigate the differential expression of the transcriptome between patients with $\mathrm{HF}$ and normal healthy controls; however, the functions and mechanisms of the identified differences were not further elucidated. In the present study, differentially expressed genes (DEGs) in the dataset GSE84796 were also identified and subsequently, the biofunction and regulatory mechanisms of these DEGs were further predicted and validated in clinical samples and cell line experiments. These findings may aid in providing novel information that will aid in understanding and treating HF.

\section{Materials and methods}

Data sourcing. The dataset GSE84796 was downloaded from the GEO database (https://www.ncbi.nlm.nih.gov/geo). This dataset comprised the gene expression data of the left ventricular tissue of 10 end-stage patients with HF that had undergone heart transplantation and the left ventricular tissue of seven healthy hearts from organ donors. Samples included in this dataset were sequenced using the GPL14550 Agilent-028004 SurePrint G3 Human GE 8x60K Microarray (Agilent Technologies, Inc.) platform.

Identification of DEGs. Raw data from the dataset was downloaded and DEGs were identified between the patients with HF and healthy controls using the limma version 3.10.3 package (http://www.bioconductor.org/packages $/ 2.9 / \mathrm{bioc} / \mathrm{html} / \mathrm{limma} . \mathrm{html}$ ) of $\mathrm{R}$ software (14), including background correction and expression value normalization. According to the annotation files, probes were mapped to gene symbols and probes that did not map to gene symbols were removed. For multiple probes mapped to one gene symbol, the average expression value was calculated as the expression level of this gene. According to the expression matrix, DEGs between the disease and control group were isolated using Bayes method in the limma package (15) with adjusted cut-off thresholds of $\mathrm{P}<0.05$ and $\log \mid$ fold change $(\mathrm{FC}) \mid>2$.

Pathway enrichment analysis for DEGs. DEGs were then subjected to signaling pathway enrichment analysis using the Kyoto Encylopedia of Genes and Genomes (KEGG) database (16). Significantly enriched DEGs in KEGG pathways were analyzed using the The Database for Annotation, Visualization and Integrated Discovery (DAVID) online tool (version 6.8; https://david-d.ncifcrf.gov) (17) using the following criteria: Enriched gene number $\geq 2$ and $\mathrm{P}<0.05$.

Protein-protein interaction (PPI) network construction. A PPI network of the DEGs was constructed using the Search Tool for the Retrieval of Interacting Genes/Proteins (STRING; version 10.0; http://string-db.org) (18), with a threshold combined score of $>0$.9. Cytoscape (version 3.2.0) (19) was used to visualize the PPI network. In addition, the plug-in MCODE (version 1.4.2; http://apps.cytoscape.org/apps/MCODE) (20) in Cytoscape was used to identify and isolate the significantly enriched modules with scores of $\geq 10$. In addition, pathway enrichment analysis for significantly enriched modules was also performed based on the information provided by KEGG pathway analysis.

Construction of TF/miRNA-target regulatory network. Using the WEB-based Gene SeT AnaLysis Tookit (WebGestalt; http://www.webgestalt.org/option.php) (21), the regulatory relationships between TF/miRNA and DEGs identified in the PPI network were predicted and visualized using Cytoscape software to obtain the TF/miRNA-target regulatory network with a threshold value of $\mathrm{P}<0.05$.

Patient studies. To further validate the results of the present study, a total of 20 patients ( 15 males and 5 females; age range, $38-52$ years; mean age $43.54 \pm 5.72$ years) with $\mathrm{HF}$ and 20 matched healthy controls (14 males and 6 females; age range $36-58$ years; mean age $44.48 \pm 6.63$ years) were enrolled at Gansu Provincial Hospital in the current study from December 2017 to May 2018. Patients in this study were examined for significant symptoms of HF and had no history of myocardial infarction or revascularization. Patients were excluded if they presented with the following: Previous stroke, diabetes mellitus, blood disease, obvious disease, infectious diseases or receiving hormone treatment. Among these participants, $5 \mathrm{ml}$ blood samples were collected to confirm the expression levels of G-protein coupled receptor 18 (GPR18), miR-155 and E26 transformation-specific transcription factor 2 (ETS2). This study was approved by the Ethic Committee of Gansu Provincial Hospital and signed informed consent was obtained from all participants.

Cell culture and transfection. The cardiomyoblast H9c2 (2-1) cell line was purchased from the American Type Culture Collection. Cells were cultured in DMEM (Gibco; Thermo Fisher Scientific, Inc.), supplemented with $10 \%$ FBS (Gibco; Thermo Fisher Scientific, Inc.), $100 \mu \mathrm{g} / \mathrm{ml}$ streptomycin (Sigma-Aldrich; Merck KGaA) and $100 \mu \mathrm{g} / \mathrm{ml}$ penicillin (Sigma-Aldrich; Merck KGaA), and maintained in a humidified atmosphere with $5 \% \mathrm{CO}_{2}$ at $37^{\circ} \mathrm{C}$.

For transfections, $1 \times 10^{5} \mathrm{H} 9 \mathrm{c} 2(2-1)$ cells/well were seeded into six-well plates and cultured overnight at $37^{\circ} \mathrm{C}$ with $70 \%$ confluency, and subsequently transfected with miR-155 mimic (50 nM; 5'-UUAAUGCUAAUCGUGAUAGGGGUC CCUAUCACGAUUAGCAUUAAUU-3'), miR-155 inhibitor (50 nM; 5'-ACCCCUAUCACGAUUAGCAUUAA-3'), negative control (NC; $50 \mathrm{nM}$; 5'-UUCUCCGAACGUGUCACG UTT-3'), pcDNA3.0-ETS2 overexpression plasmid, an empty pcDNA3.0 plasmid as the NC (100 nM; pcDNA3.0), small interfering RNA (si) targeting ETS2 (si-ETS2; $100 \mathrm{~nm}$; sense, 5'-GGGAACAUCUGGAGCAAAUTT-3'; antisense, 5'-AUU UGCUCCAGAUGUUCCCTT-3') or si-NC (100 nM; sense, 5'-UUCUCCGAACGUGUCACGUTT-3'; antisense, 5'ACG UGACACGUUCGGAGAATT-3') using Lipofectamine ${ }^{\circledR} 2000$ reagent (Thermo Fisher Scientific, Inc.), according to the manufacturer's protocol. miR-155 mimic, miR-155 inhibitor, miRNA negative control, pcDNA3.0, pcDNA3.0-ETS2, si-NC and si-ETS2 were purchased from Nanjing KeyGen Biotech Co., Ltd. Cells were transfected for $48 \mathrm{~h}$ prior to subsequent experimentation.

Cell viability. Cell viability was determined using a Cell Counting Kit-8 assay (CCK-8; Dojindo Molecular 

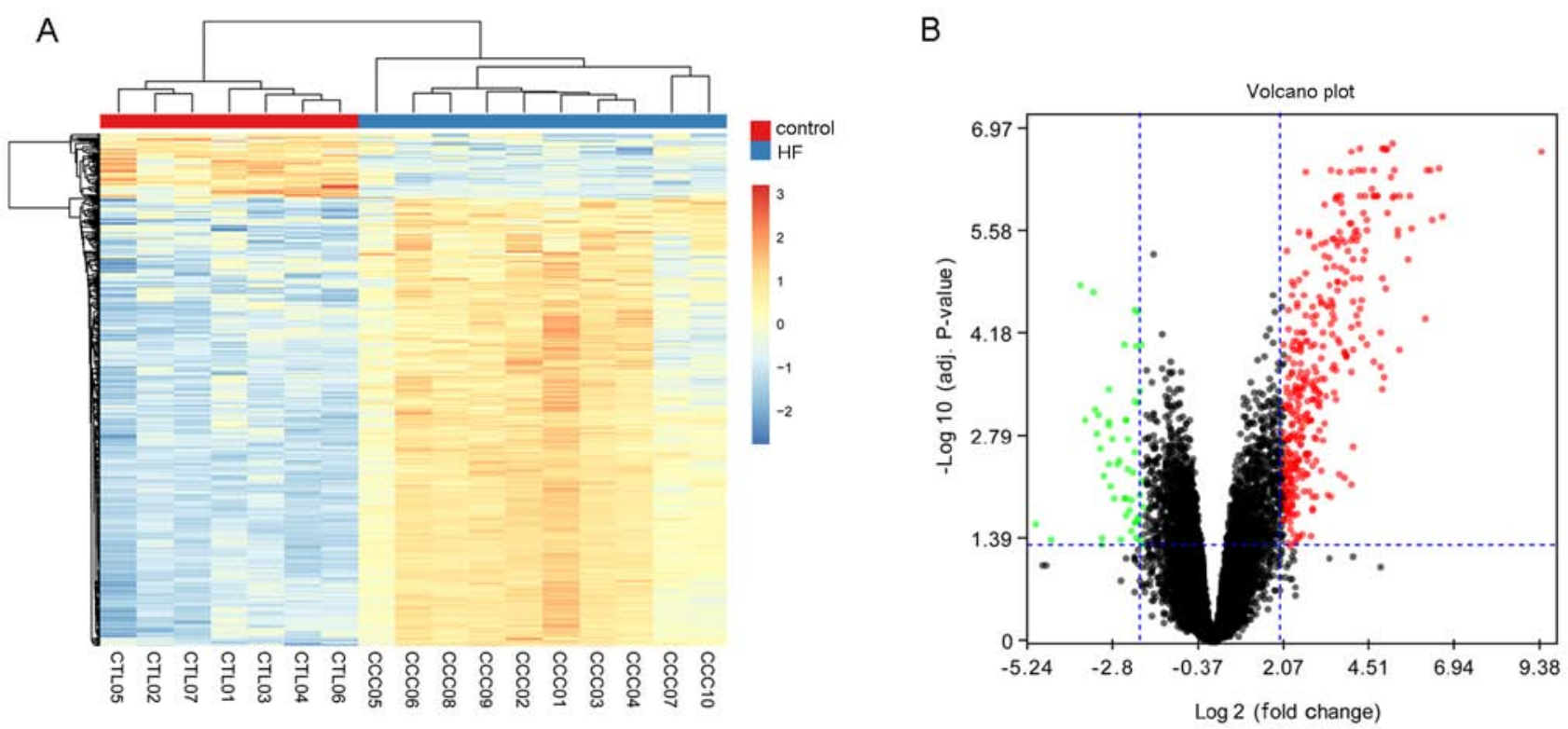

Figure 1. Heatmap and volcano plot for differentially expressed genes identified in HF. (A) Heatmap and (B) volcano plot. Red columns in the heatmap indicate higher gene expression, while blue columns in the heatmap indicate lower gene expression. For the volcano plot, red plots represent upregulated DEGs, green plots represent downregulated DEGs and black plots represent non-differentially expressed genes. HF, heart failure; DEG, differentially expressed genes.

Technologies, Inc.), according to the manufacturer's protocol. Briefly, 2x10 4 Hc2 (2-1) cells/well were plated into 96-well plates and incubated in a humidified atmosphere with $5 \%$ $\mathrm{CO}_{2}$ at $37^{\circ} \mathrm{C}$. Following incubation for 24,48 and $72 \mathrm{~h}, 10 \mu \mathrm{l}$ CCK-8 solution was added to each well and cultured for $1 \mathrm{~h}$ at $37^{\circ} \mathrm{C}$. The optical density of each well at $450 \mathrm{~nm}$ was recorded using a Varioskan Flash microplate reader (Thermo Fisher Scientific, Inc.).

Flow cytometric analysis of apoptosis. Briefly, a total $1 \times 10^{5}$ of H9c2 (2-1) cells were seeded on a six-well plate and incubated in a humidified atmosphere with $5 \% \mathrm{CO}_{2}$ at $37^{\circ} \mathrm{C}$ with $70 \%$ confluency overnight. Following transfection and incubation for $48 \mathrm{~h}$, the rate of apoptosis was determined using the Annexin V-FITC Apoptosis Detection kit (BioVision, Inc.), according to the manufacturer's protocol. Apoptotic cells were analyzed using a BD FACScan ${ }^{\mathrm{TM}}$ flow cytometer (BD Biosciences).

Reverse transcription-quantitative PCR (RT-qPCR). Total RNA was extracted from tissue and cell lines using TRIzol ${ }^{\mathrm{TM}}$ reagent (Invitrogen; Thermo Fisher Scientific, Inc.), according to the manufacturer's protocol. To determine mRNA expression levels, $2 \mu \mathrm{g}$ RNA was reversed transcribed into cDNA using the PrimeScript RT Master mix (Takara Biotechnology Co., Ltd.) according to the manufacturer's protocol. qPCR was subsequently performed using the SYBR ${ }^{\circledR}$ Green PCR Master mix (Thermo Fisher Scientific Inc.) according the manufacturer's protocol. The following primer pairs were used for the qPCR: GPR18 forward, 5'-CCACCAAGAAGAGAACCA C-3' and reverse, 5'-GAAGGGCATAAAGCAGACG-3'; ETS2 forward, 5'-GTGGACCTATTCAGCTGTGG-3' and reverse, 5'-TTCCCCGACGTCTTGTGGAT-3'; and $\beta$-actin forward, 5'-CTGGGACGACATGGAGAAAA-3' and reverse, 5'-AAG GAAGGCTGGAAGAGTGC-3'. The following thermocycling conditions were used for the qPCR: $50^{\circ} \mathrm{C}$ for $3 \mathrm{~min} ; 95^{\circ} \mathrm{C}$ for
$3 \mathrm{~min}$; and 40 cycles of $95^{\circ} \mathrm{C}$ for $10 \mathrm{sec}$ and $60^{\circ} \mathrm{C}$ for $30 \mathrm{sec}$. $\beta$-actin was used as the internal loading control.

For miRNA, $2 \mu \mathrm{g}$ total RNA was reversed transcribed into cDNA using the TaqMan MicroRNA Reverse Transcription kit according to the manufacturer's protocol (Applied Biosystems; Thermo Fisher Scientific, Inc.). qPCR was performed using SYBR ${ }^{\circledR}$ Green PCR Master mix (Thermo Fisher Scientific, Inc.) according to the manufacturer's protocol. The following primer pairs were used for the qPCR: miR-155 forward, 5'-GCGGTTAATGCTAATCGT GAT-3' and reverse, 5'-GTGCAGGGTCCGAGGT-3'; and U6 forward, 5'-CTCGCTTCGGCAGCACA-3' and reverse, 5'-AACGCTTCACGAATTTGCGT-3'. The following thermocycling conditions were used for the qPCR: $95^{\circ} \mathrm{C}$ for $10 \mathrm{~min}$; and 40 cycles of $95^{\circ} \mathrm{C}$ for $15 \mathrm{sec}, 60^{\circ} \mathrm{C}$ for $30 \mathrm{sec}$ and $60^{\circ} \mathrm{C}$ for $1 \mathrm{~min}$. U6 was used as the internal loading control for the miRNA. mRNA and miRNA expression levels were quantified using the $2^{-\Delta \Delta \mathrm{Cq}}$ method (22).

Western blot analysis. Total protein was extracted from cells using RIPA lysis buffer containing a protease inhibitor cocktail (Roche Diagnostics $\mathrm{GmbH}$ ). Total protein was quantified using a BCA assay kit (Pierce; Thermo Fisher Scientific, Inc.) and $25 \mu \mathrm{g}$ protein/lane was separated using $10 \%$ SDS-PAGE. The separated proteins were subsequently transferred onto PVDF membranes (GE Healthcare) and blocked for $1 \mathrm{~h}$ at room temperature with $5 \%$ non-fat milk. The membranes were incubated with primary antibodies against GPR18 (1:2,000; cat. no. PA5-23218; Invitrogen; Thermo Fisher Scientific, Inc.) and $\beta$-actin (1:5,000; cat. no. sc-69879; Santa Cruz Biotechnology, Inc.) at $4^{\circ} \mathrm{C}$ overnight. Following the primary antibody incubation, membranes were incubated with horseradish peroxidase-labeled goat anti-rabbit IgG (1:5,000; cat. no. sc-2030; Santa Cruz Biotechnology, Inc.) and goat anti-mouse IgG secondary antibodies (1:5,000; cat. no. sc-2005; Santa Cruz Biotechnology, Inc.) at room temperature for $1 \mathrm{~h}$. Protein bands were visualized 
Table I. Top Kyoto Encyclopedia of Genes and Genomes pathways enriched by differentially expressed genes.

\begin{tabular}{llcc}
\hline Pathway ID & \multicolumn{1}{c}{ Pathway name } & Count & P-value \\
\hline hsa04060 & Cytokine-cytokine receptor interaction & 31 & $3.46 \times 10^{-16}$ \\
hsa04650 & Natural killer cell mediated cytotoxicity & 22 & $6.05 \times 10^{-14}$ \\
hsa05340 & Primary immunodeficiency & 14 & $6.77 \times 10^{-14}$ \\
hsa04660 & T cell receptor signaling pathway & 19 & $3.28 \times 10^{-12}$ \\
hsa04640 & Hematopoietic cell lineage & 16 & $2.02 \times 10^{-10}$ \\
hsa04514 & Cell adhesion molecules & 18 & $6.15 \times 10^{-9}$ \\
hsa04062 & Chemokine signaling pathway & 20 & $1.08 \times 10^{-8}$ \\
hsa04064 & NF-KB signaling pathway & 13 & $2.47 \times 10^{-7}$ \\
hsa05323 & Rheumatoid arthritis & 13 & $2.81 \times 10^{-7}$ \\
hsa04672 & Intestinal immune network for IgA production & 10 & $4.78 \times 10^{-7}$ \\
\hline
\end{tabular}

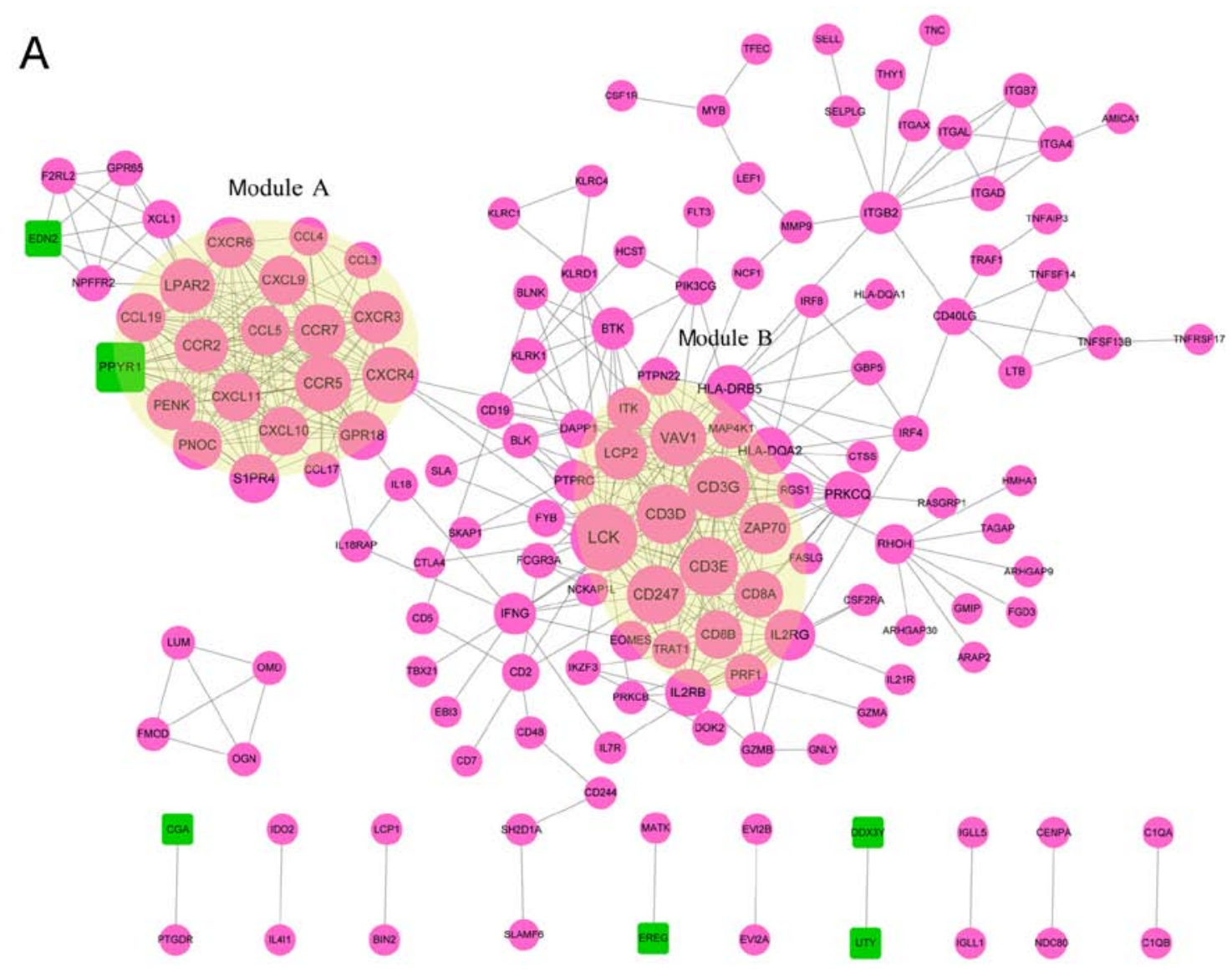

B Module A

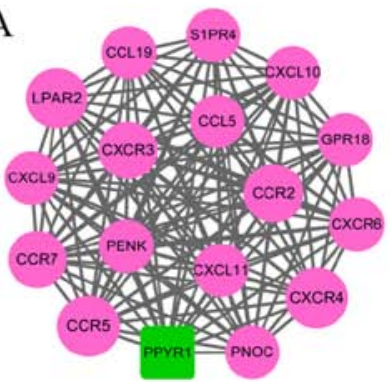

C Module B

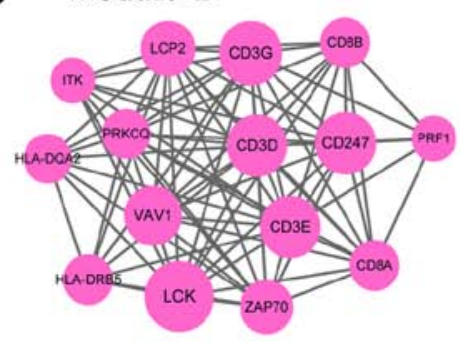

Figure 2. PPI network for DEG-encoded proteins and Module Analysis. (A) PPI network for Module A and B. (B) A total of 17 nodes and 136 relationship pairs with a score of 12 were identified in Module A. (C) A total of 15 nodes and 84 relationship pairs were identified in Module B. Pink circles represent upregulated DEG-encoded proteins, green squares represent downregulated DEG-encoded proteins, and the yellow area represents isolated modules in the network. PPI, protein-protein interaction; DEGs, differentially expressed genes. 
Table II. Kyoto Encyclopedia Genes and Genomes pathway enrichment analysis results for Module A and Module B.

A, Module A

\begin{tabular}{|c|c|c|c|c|}
\hline Pathway ID & Pathway name & Count & P-value & Proteins \\
\hline hsa04062 & Chemokine signaling pathway & 11 & $9.85 \times 10^{-15}$ & $\begin{array}{l}\text { CCR7, CCR5, CXCR4, CCR2, CXCR6, } \\
\text { CXCL9, CCL19, CXCR3, CCL5, } \\
\text { CXCL11, CXCL10 }\end{array}$ \\
\hline hsa04060 & Cytokine-cytokine receptor interaction & 11 & $8.58 \times 10^{-14}$ & $\begin{array}{l}\text { CCR7, CCR5, CXCR4, CCR2, CXCR6, } \\
\text { CXCL9, CCL19, CXCR3, CCL5, } \\
\text { CXCL11, CXCL10 }\end{array}$ \\
\hline hsa04620 & Toll-like receptor signaling pathway & 4 & $6.98 \times 10^{-04}$ & CXCL9, CCL5, CXCL11, CXCL10 \\
\hline
\end{tabular}

B, Module B

\begin{tabular}{|c|c|c|c|c|}
\hline Pathway ID & Pathway name & Count & P-value & Proteins \\
\hline hsa04660 & T cell receptor signaling pathway & 12 & $1.64 \times 10^{-18}$ & $\begin{array}{l}\text { ITK, PRKCQ, CD3G, CD3D, CD8A, } \\
\text { CD3E, CD8B, CD247, LCK, ZAP70, } \\
\text { VAV1, LCP2 }\end{array}$ \\
\hline hsa05340 & Primary immunodeficiency & 6 & $4.12 \times 10^{-9}$ & $\begin{array}{l}\text { CD3D, CD8A, CD3E, CD8B, LCK, } \\
\text { ZAP70 }\end{array}$ \\
\hline hsa04640 & Hematopoietic cell lineage & 6 & $4.59 \times 10^{-7}$ & $\begin{array}{l}\text { CD3G, CD3D, CD8A, CD3E, CD8B, } \\
\text { HLA-DRB5 }\end{array}$ \\
\hline hsa04650 & Natural killer cell mediated cytotoxicity & 6 & $2.79 \times 10^{-6}$ & $\begin{array}{l}\text { PRF1, CD247, LCK, ZAP70, VAV1, } \\
\text { LCP2 }\end{array}$ \\
\hline hsa05166 & HTLV-I infection & 6 & $1.02 \times 10^{-4}$ & $\begin{array}{l}\text { CD3G, CD3D, CD3E, LCK, } \\
\text { HLA-DRB5, HLA-DQA2 }\end{array}$ \\
\hline hsa04612 & Antigen processing and presentation & 4 & $4.27 \times 10^{-4}$ & $\begin{array}{l}\text { CD8A, CD8B, HLA-DRB5, } \\
\text { HLA-DQA2 }\end{array}$ \\
\hline hsa05142 & Chagas disease (American trypanosomiasis) & 4 & $1.07 \times 10^{-3}$ & CD3G, CD3D, CD3E, CD247 \\
\hline hsa05332 & Graft-versus-host disease & 3 & $1.94 \times 10^{-3}$ & PRF1, HLA-DRB5, HLA-DQA2 \\
\hline hsa05162 & Measles & 4 & $2.17 \times 10^{-3}$ & PRKCQ, CD3G, CD3D, CD3E \\
\hline hsa05330 & Allograft rejection & 3 & $2.44 \times 10^{-3}$ & PRF1, HLA-DRB5, HLA-DQA2 \\
\hline hsa04514 & Cell adhesion molecules (CAMs) & 4 & $2.62 \times 10^{-3}$ & $\begin{array}{l}\text { CD8A, CD8B, HLA-DRB5, } \\
\text { HLA-DQA2 }\end{array}$ \\
\hline hsa04940 & Type I diabetes mellitus & 3 & $3.13 \times 10^{-3}$ & PRF1, HLA-DRB5, HLA-DQA2 \\
\hline hsa05320 & Autoimmune thyroid disease & 3 & $4.77 \times 10^{-3}$ & PRF1, HLA-DRB5, HLA-DQA2 \\
\hline hsa05416 & Viral myocarditis & 3 & $5.71 \times 10^{-3}$ & PRF1, HLA-DRB5, HLA-DQA2 \\
\hline hsa04064 & NF-kappa B signaling pathway & 3 & $1.29 \times 10^{-2}$ & PRKCQ, LCK, ZAP70 \\
\hline
\end{tabular}

using an ECL plus kit (GE Healthcare) and analyzed with ChemiDoc XRS+ luminescent image analyzer (Bio-Rad Laboratories, Inc.).

Statistical analysis. Statistical analysis was performed using GraphPad Prism 6.0 software (GraphPad Software, Inc.) and data are presented as the mean \pm standard deviation of three experimental repeats. Statistical differences between 2 groups were determined using Student's t-tests, whereas differences between $>2$ groups were determined using one-way ANOVA, with Tukey's post hoc test for multiple comparisons. $\mathrm{P}<0.05$ was considered to indicate a statistically significant difference.

\section{Results}

DEGs screening. After preprocessing, a total of 419 DEGs were identified, including 366 upregulated genes and 53 downregulated genes, which suggested that DEGs could distinguish patients with HF from healthy controls. The bi-clustering of identified DEGs are presented in Fig. 1A. and the volcano plot of DEGs is presented in Fig. 1B.

Functional enrichment of DEGs. To further identify the molecular mechanisms that the DEGs participated within, KEGG pathway enrichment analysis for upregulated and downregulated DEGs was conducted using the DAVID online 


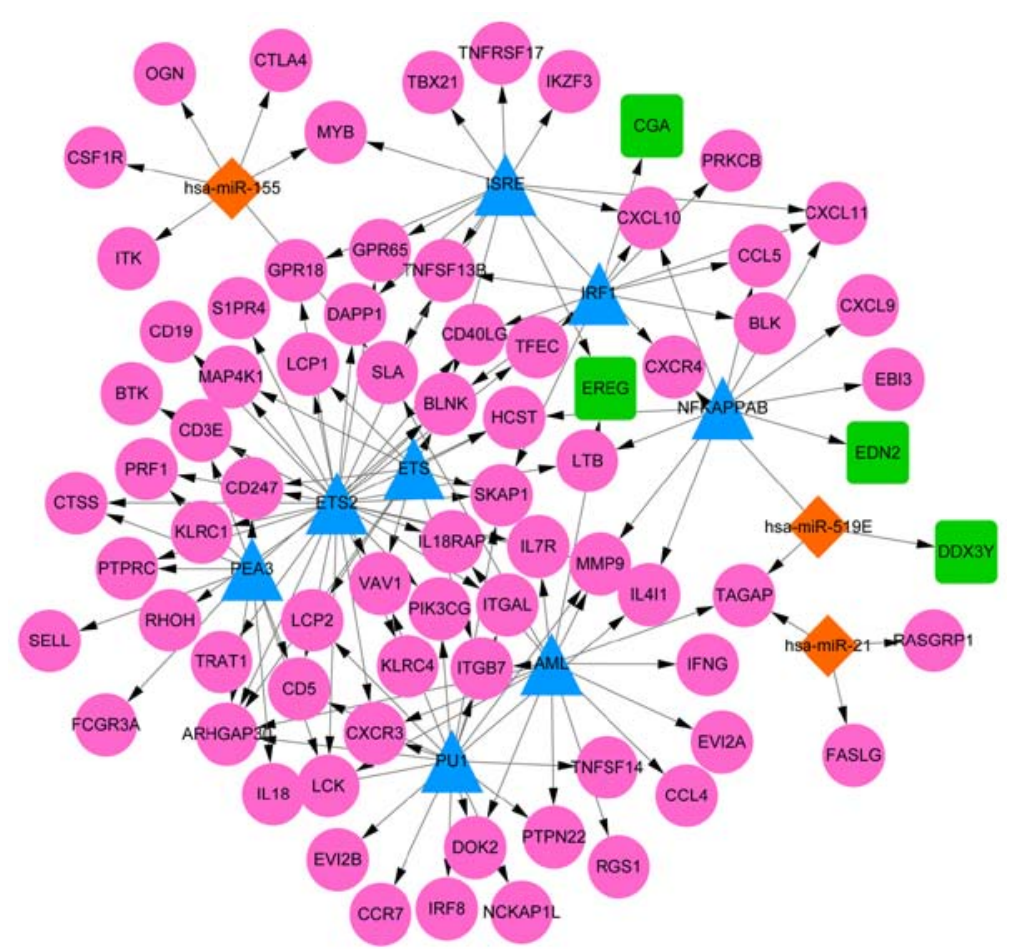

Figure 3. TF/miRNA-target regulatory network in heart failure. Blue triangle represents TFs, the orange diamond represents miRNA, the pink circles represents the upregulated DEGs and the green square represents the downregulated DEGs. TF, transcription factor; miRNA, microRNA; DEGs, differentially expressed genes.

tool. Due to the low number of downregulated DEGs identified, no KEGG pathway was indicated to be significantly enriched by the downregulated DEGs; however, 40 KEGG pathways were enriched by the upregulated DEGs, including 'cytokine-cytokine receptor interaction' $\left(\mathrm{P}=3.46 \times 10^{-16}\right)$, 'natural killer cell mediated cytotoxicity' $\left(\mathrm{P}=6.05 \times 10^{-14}\right)$ and 'primary immunodeficiency' $\left(\mathrm{P}=6.77 \times 10^{-14}\right)$. The top 10 pathways are presented in Table I.

PPI network and model analysis. According to the STRING database, the PPI network for DEG-encoded proteins was constructed with 148 nodes and 482 edges (Fig. 2A). The top 10 nodes that were expressed to a higher degree were: Lymphocyte-specific protein tyrosine kinase (LCK), cluster of differentiation $3 \mathrm{~g}(\mathrm{CD} 3 \mathrm{G})$, cluster of differentiation $3 \mathrm{e}$ (CD3E), cluster of differentiation 3d (CD3D), cluster of differentiation 247 (CD247), van guanine nucleotide exchange factor 1 (VAV1), C-C motif chemokine receptor 5 (CCR5), C-X-C motif chemokine receptor 4 (CXCR4), lysophosphatidic acid receptor (LPAR2) and C-C motif chemokine receptor 2 (CCR2). Two significantly enriched modules with an enriched score $\geq 10$ were isolated from the PPI network. Module A contained 17 nodes and 136 relationship pairs with a score of 12 (Fig. 2B), which were significantly enriched in the 'chemokine signaling pathway' $\left(\mathrm{P}=9.85 \times 10^{-15}\right)$, 'cytokine-cytokine receptor interaction' $\left(\mathrm{P}=8.58 \times 10^{-14}\right)$ and 'Toll-like receptor signaling pathway' $\left(\mathrm{P}=6.98 \times 10^{-4}\right.$; Table II). A total of 15 nodes and 84 relationship pairs were included in module B with a score of 12 (Fig. 2C), and this module was significantly enriched in 15 KEGG pathways, including ' $T$ cell receptor signaling pathway' $\left(\mathrm{P}=1.64 \times 10^{-18}\right)$, 'primary immunodeficiency'
$\left(\mathrm{P}=4.12 \times 10^{-9}\right)$ and 'hematopoietic cell lineage' $\left(\mathrm{P}=4.59 \times 10^{-7}\right.$; Table II).

TF/miRNA-target regulatory network. According to the WebGestalt database, among 10 nodes in this network, there were $8 \mathrm{TFs}$ identified to target the DEGs identified in the current study, including ETS2, pullulanase 1 (PU1), acute myeloid leukemia protein (AML), interferon-stimulated response elements (ISRE), polyomavirus enhancer activator 3 (PEA3), interferon 1 (IRF1), nuclear factor kappa B (NFKAPPAB) and E26 transformation-specific transcription factor (ETS). Moreover, a total of three miRNAs were predicted to target DEGs: miR-155, miR-21 and miR-519. Through combining the regulatory relationships of TF/gene and miRNA/gene, the TF/miRNA-target regulatory network was constructed (Fig. 3), which included 86 nodes (71 upregulated DEGs and 4 downregulated DEGs) and 138 relationship pairs.

Validation of gene expression in clinical samples. To confirm the findings of the bioinformatics analysis, the expression levels of miR-155, GPR18 and ETS2 were determined in patients with HF. The expression levels of GPR18 and ETS2 were significantly increased in HF samples compared with the healthy controls $(\mathrm{P}<0.0001$; Fig. $4 \mathrm{~A}$ and $\mathrm{C})$, whereas the expression levels of miR-155 were significantly decreased in HF samples compared with the healthy controls $(\mathrm{P}<0.0001$; Fig. 4B). These results were consistent with the findings observed using bioinformatics.

ETS2 is a TF for GPR18. To further confirm the regulation of the upregulated expression of GPR18, the expression levels of ETS2 were verified and the expression levels of GPR18 were 
A

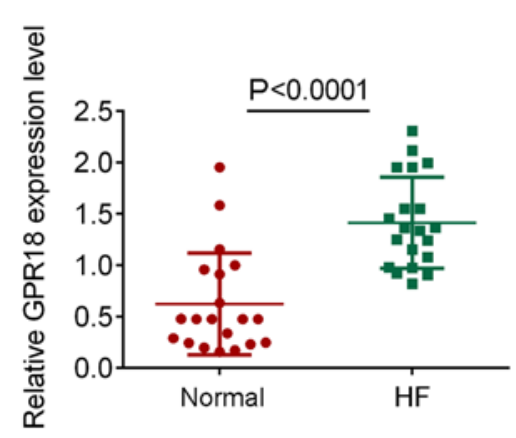

C

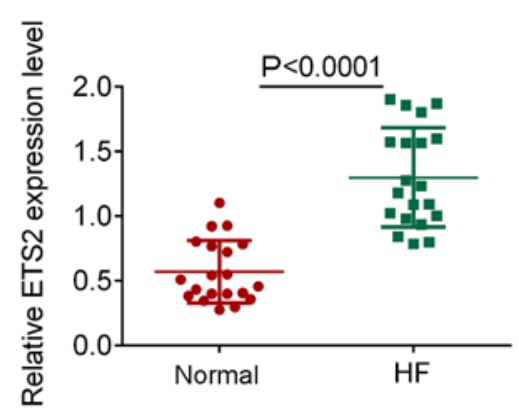

B

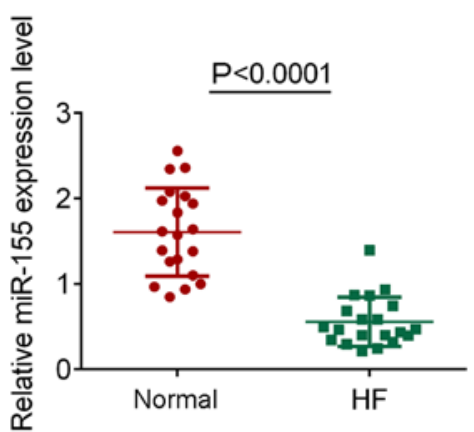

Figure 4. mRNA expression levels of GPR18, miR-155 and ETS2 in clinical patients with HF. Expression levels of (A) GPR18, (B) miR-155 and (C) ETS2 in patients with HF compared with normal tissue was assessed using reverse transcription-quantitative PCR. Normal control, $\mathrm{n}=20$; patients with $\mathrm{HF}$, $\mathrm{n}=20$. miR, microRNA; GPR18, G-protein coupled receptor 18; ETS2, E26 transformation-specific transcription factor 2.
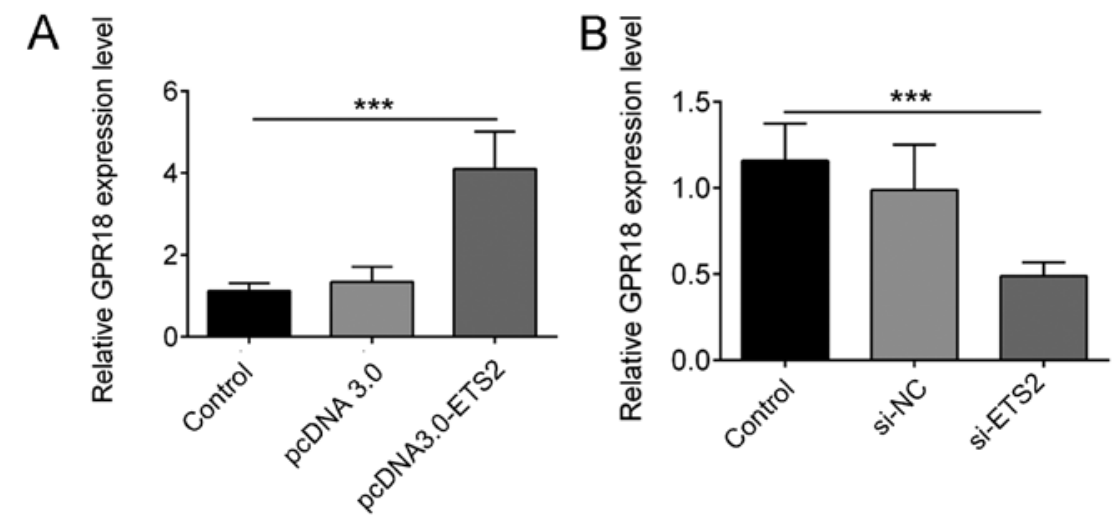

C

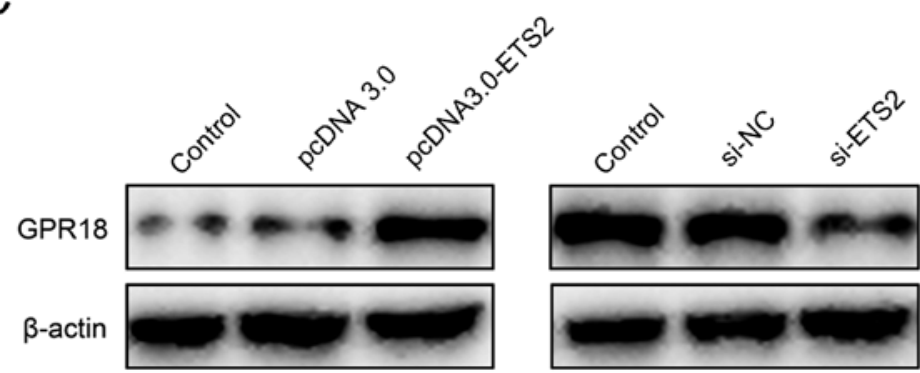

Figure 5. GPR18 is a target gene of the transcription factor ETS2. (A) mRNA expression levels of GPR18 were determined following the transfection of H9c2 (2-1) cells with an ETS2 overexpressing plasmid. (B) mRNA expression levels of GPR18 were determined following the transfection of H9c2 (2-1) cells with si-ETS2. (C) Protein expression levels of GPR18 following the transfection of cells with either ETS2 overexpression plasmid or si-ETS2. ${ }^{* * *}$ P $<0.001$ vs. control group. GPR18, G-protein coupled receptor 18; si, small interfering RNA; ETS2, E26 transformation-specific transcription factor 2; NC, negative control.

determined at the cellular level following the use of ETS2 overexpression plasmids or siRNA targeting ETS2, of which their transfection was proved to be successful (Fig. S1A-D). It was demonstrated that the overexpression of ETS2 with the pcDNA3.0-ETS2 plasmid could significantly increase the mRNA and protein expression levels of GPR18 compared with the control group $(\mathrm{P}<0.001$; Fig. $5 \mathrm{~A}$ and $\mathrm{C})$, whereas the genetic silencing of ETS2 with si-ETS2 could significantly decrease the expression levels of GPR18 in H9c2 (2-1) cells compared with the control group ( $\mathrm{P}<0.001$; Fig. 5B and $\mathrm{C})$. 
A

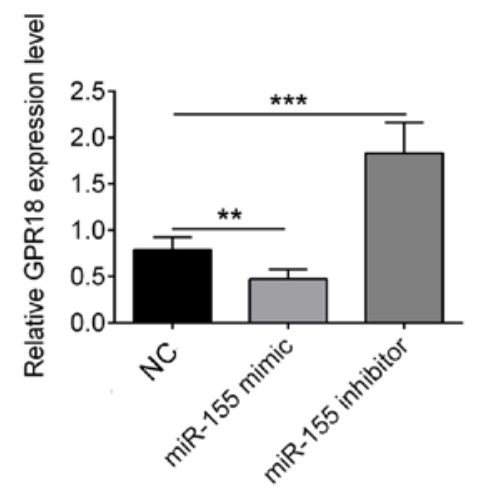

C

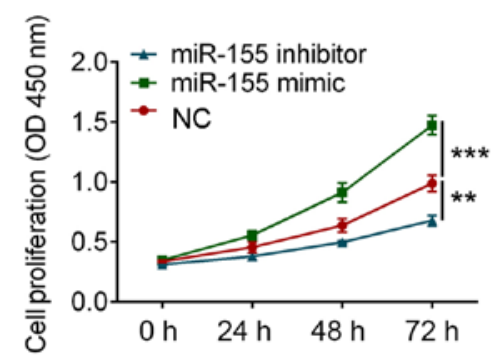

B
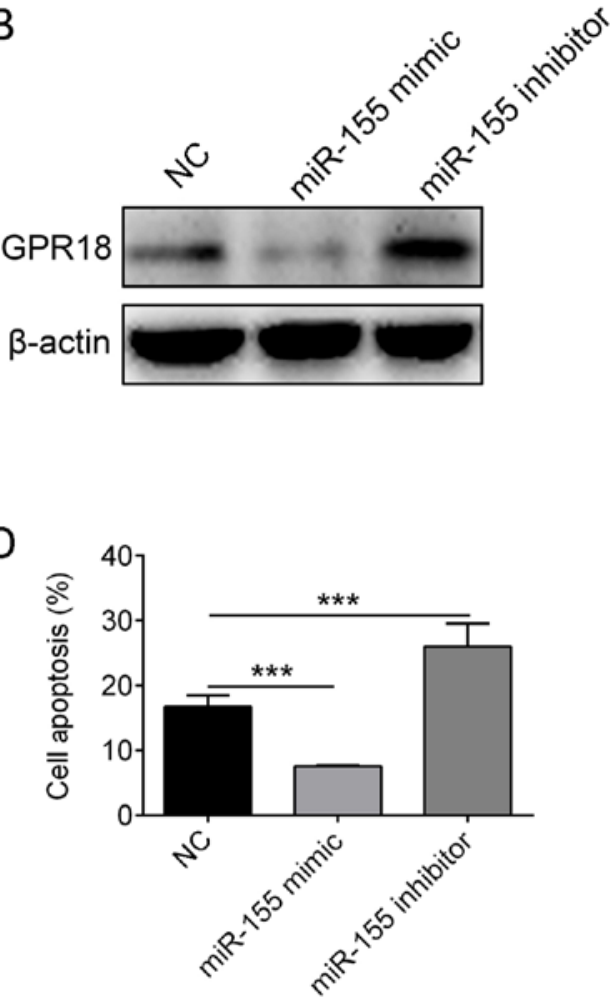

E

NC

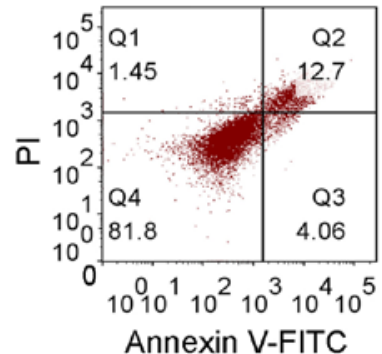

$\bar{\alpha}$

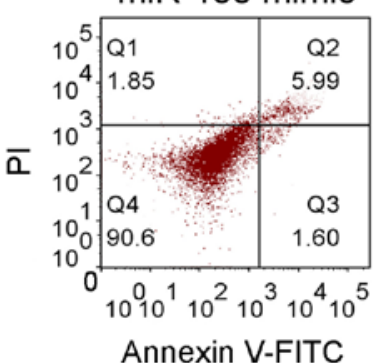

miR-155 inhibitor

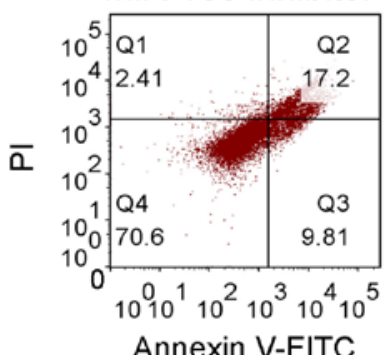

Figure 6. miR-155 regulates cell viability and apoptosis through targeting GPR18 in H9c2 (2-1) cells. (A) mRNA expression levels of GPR18 were determined using reverse transcription-quantitative PCR following the overexpression and silencing of miR-155 using a mimic and inhibitor, respectively in H9c2 (2-1) cells. (B) Protein expression levels of GPR18 were determined using western blotting following the overexpression and silencing of miR-155 using a mimic and inhibitor, respectively in H9c2 (2-1) cells. (C) Cell viability of H9c2 (2-1) cells. (D) Semi-quantification of apoptosis in H9c2 (2-1) cells (E) Apoptosis in H9c2 (2-1) cells. ${ }^{* *} \mathrm{P}<0.01$ and ${ }^{* * *} \mathrm{P}<0.001$ vs. control group. NC, negative control; miR, microRNA; GPR18, G-protein coupled receptor 18.

These findings indicated that GPR18 may be a downstream target of ETS2, and ETS2 may target GPR18 to promote the progression of HF.

miR-155 regulates the cell viability and apoptosis of H9c2 (2-1) through GPR18. The regulatory relationship between GPR18 and miR-155 was also verified at the cellular level following the use of miR-155 mimics or miR-155 inhibitors, of which their transfection was proved to be successful (Fig. S1E). The results revealed that the overexpression of miR-155 mimic decreased the expression levels of GPR18 $(\mathrm{P}<0.01)$, whereas the miR-155 inhibitor increased the expression levels of GPR18 in H9c2 (2-1) cells compared with the control group $(\mathrm{P}<0.001$; Fig. 6A and $\mathrm{B})$. The increased expression of miR-155 was also indicated to significantly increase the cell viability in $\mathrm{H} 9 \mathrm{c} 2(2-1)$ cells $(\mathrm{P}<0.01)$, but attenuated apoptosis compared with the control group $(\mathrm{P}<0.001)$, whereas the decreased expression of miR-155 using miR-155 inhibitor demonstrated opposite effects to the miR-155 mimic-transfected cells $(\mathrm{P}<0.001$; Fig. 6C-E). These findings indicated that miR-155 might inhibit the apoptosis of H9c2 (2-1) through downregulating the expression of GPR18.

\section{Discussion}

HF is a common clinical syndrome worldwide characterized by heart structure damage and/or heart dysfunction, resulting in fatigue and dyspnea at rest (23). HF is a multifactorial disease and its development is associated with a complex and sophisticated regulation, but the exact mechanism remains to be elucidated (24). Although numerous researches have been performed $(25,26)$, the exact mechanism of HF remains to be elucidated. In the present study, a total of 419 DEGs were identified and their encoding proteins were used to construct a PPI network, and GPR18 was identified as a hub node in this network. Further bioinformatics and 
experimental analyses showed that GPR 18 could be targeted by miR-155 and the TF ETS2, suggesting that miR-155 and ETS2 might play critical role in the development of HF via targeting GPR18.

GPR18 is a deorphaned lipid receptor that can be activated by behaviorally inactive atypical cannabinoid and N-arachidonoyl glycine (27). A number of tissues have reportedly demonstrated very low expression of GPR18, including the brain, heart, liver, lung and ovaries (28). A previous study identified that GPR 18 serves a critical role in modulating cardiovascular function (24) and Penumarti and Abdel-Rahman (29) reported that GPR18 mediated hypertension through activating the $\mathrm{PI} 3 \mathrm{~K} / \mathrm{AKT} / \mathrm{ERK} / \mathrm{nNOS}$ signaling pathway and suppressing cAMP in an animal model (30). However, the function of GPR18 in HF is relatively unreported. In the present study, GPR18 expression levels were significantly increased in patients with HF compared with healthy controls, which was further validated with clinical studies; GPR18 expression was significantly elevated in patients with HF compared with healthy controls. All of these findings indicated that GPR18 may serve a role in promoting the pathogenesis of HF.

ETS2, encoded by E26 oncogene homolog 2, serves an important role in the pathogenesis of cardiovascular disease (31). Previous studies have demonstrated that ETS2 and Mesp1 are two important TFs involved in the reprogramming of fibroblasts into cardiac progenitor-like cells $(32,33)$. In addition, Rowell et al (34) revealed that reduced ETS2 expression in endothelial progenitor cells was a beneficial biomarker of sitagliptin efficacy in patients undergoing coronary artery-bypass grafting. The current study also revealed that ETS family members, including ETS-related transcription factor 1 (Elf1) and ETS2, were upregulated in late-HF (34). In the present study, ETS2 expression levels were found to be significantly increased in patients with HF compared with the healthy controls. Moreover, the overexpression of ETS2 significantly increased the expression levels of GPR18, whereas the silencing of ETS2 expression significantly reduced the expression levels of GPR18 in H9c2 (2-1) cells. These results indicated that GPR18 may be a downstream target for ETS2. Considering this evidence, it was hypothesized that ETS2 may promote the pathogenesis of HF through targeting and regulating GPR18 expression.

miR-155 is an established miRNA that is involved in the regulation of a number of different cancer pathways, as well as in cardiovascular diseases (35). Bao et al (36) demonstrated that miR-155 and miR-148a could reduce cardiac injury through suppressing $\mathrm{NF}-\kappa \mathrm{B}$ expression in acute viral myocarditis. Moreover, miR-155 was demonstrated to serve as a potential marker for arrhythmic risk in patients with chronic heart failure (37) and Matsumoto et al (38) revealed that miR-155 is a risk factor for cardiac death. In the current study, miR-155 expression was significantly decreased in patients with $\mathrm{HF}$, and it was observed to promote the proliferation of $\mathrm{H} 9 \mathrm{c} 2$ (2-1) cells and reduce apoptosis in $\mathrm{H} 9 \mathrm{c} 2(2-1)$ cells through negatively regulating the expression levels of GPR18. These findings suggested that miR-155 may serve a crucial role in regulating cell viability and apoptosis in HF through targeting and regulating GPR18 expression.
There are multiple limitations associated with the current study; For example, because no relevant clinical information was provided for the GSE84796 dataset in the GEO database, investigations between the molecular mechanisms and clinical features were not analyzed in the present study. Additionally, due to time and resource limitations, an insufficient number of matched heart tissue samples were collected from patients to confirm the results of the current study. Due to this, the study used blood samples instead to perform validation studies, but further validation in clinical samples is required in the future. Additionally, due to the limited funding for the current study, only H9c2 (2-1) cell lines were used for the following experimental validations and further validation in additional human cell lines, including HCM HJ1-I, are required to further validate the results of the current study. Finally, also due to the limited funds available, further investigations into ETS2 and subsequent pathway analysis were not performed in the present study. However, despite these limitations, the findings in the present study may provide some novel insight in understanding $\mathrm{HF}$.

In conclusion, both GRP18 and ETS2 expression levels were demonstrated to be significantly increased in $\mathrm{HF}$ compared with healthy controls, whereas the expression levels of miR-155 were significantly reduced in HF compared with healthy controls. These findings suggested that GPR 18 may be targeted by EST2 and miR-155, and miR-155 may promote cell viability and suppress apoptosis in HF through targeting and regulating GPR18.

\section{Acknowledgements}

Not applicable.

\section{Funding}

No funding was received.

\section{Availability of data and materials}

The datasets used and/or analyzed during the current study are available from the corresponding author on reasonable request.

\section{Authors' contributions}

$\mathrm{XM}$ and JL designed all the experiments and revised the paper; HS, YC and YZ performed the experiments; and YC and $\mathrm{XM}$ wrote the manuscript. All authors read and approved the final manuscript.

\section{Ethics approval and consent to participate}

The present study was approved by the Ethics Committee of Gansu Provincial Hospital and informed consent was obtained from all participants.

\section{Patient consent for publication}

Not applicable. 


\section{Competing interests}

The authors declare that they have no competing interests.

\section{References}

1. Forouzanfar MH, Moran A, Phillips D, Mensah GA, Ezzati M, Naghavi M and Murray Christopher JL: Prevalence of heart failure by cause in 21 regions: global burden of diseases, injuries and risk factors-2010 study. Journal of the American College of Cardiology 61: E786-E786, 2013.

2. Farré N, Vela E, Clèries M, Bustins M, Cainzos-Achirica M, Enjuanes C, Moliner P, Ruiz S, Verdú-Rotellar JM and Comín-Colet J: Real world heart failure epidemiology and outcome: A population-based analysis of 88,195 patients. PLoS One 12: e0172745, 2017.

3. Cook C, Cole G, Asaria P, Jabbour R and Francis DP: The annual global economic burden of heart failure. Int J Cardiol 171: 368-376, 2014.

4. Inglis SC, Clark RA, Dierckx R, Prieto-Merino D and Cleland JGF: Structured telephone support or non-invasive telemonitoring for patients with heart failure. Cochrane Database Syst Rev 10: CD007228, 2015.

5. Pinti MV, Hathaway QA and Hollander JM: Role of microRNA in metabolic shift during heart failure. Am J Physiol Heart Circ Physiol 312: H33-H45, 2017.

6. Duan Q, Yang L, Gong W, Chaugai S, Wang F, Chen C, Wang P, Zou MH and Wang DW: MicroRNA-214 is upregulated in heart failure patients and suppresses XBP1-mediated endothelial cells angiogenesis. J Cell Physiol 230: 1964-1973, 2015.

7. Duan Q, Chen C, Yang L, Li N, Gong W, Li S and Wang DW: MicroRNA regulation of unfolded protein response transcription factor XBP1 in the progression of cardiac hypertrophy and heart failure in vivo. J Transl Med 13: 363, 2015.

8. Masson S, Batkai S, Beermann J, Bär C, Pfanne A, Thum S, Magnoli M, Balconi G, Nicolosi GL, Tavazzi L, et al: Circulating microRNA-132 levels improve risk prediction for heart failure hospitalization in patients with chronic heart failure. Eur J Heart Fail 20: 78-85, 2018

9. Li S, Fan Q, He S, Tang T, Liao Y and Xie J: MicroRNA-21 negatively regulates Treg cells through a TGF- $\beta 1 /$ Smad-independent pathway in patients with coronary heart disease. Cell Physiol Biochem 37: 866-878, 2015.

10. Mcculley DJ and Black BL: Chapter nine-Transcription Factor Pathways and Congenital Heart Disease. In: Current Topics in Developmental Biology. Vol 100. Elsevier Inc., 2012.

11. Bakker ML, Boink GJJ, Boukens BJ, Verkerk AO, Malou VDB, Den Haan AD, Hoogaars WM,Buermans HP, deBakkerJM,Seppen J, et al: T-box transcription factor TBX3 reprogrammes mature cardiac myocytes into pacemaker-like cells. Cardiovasc Res 94: 439-449, 2012.

12. Courties G, Heidt T, Sebas M, Iwamoto Y, Jeon D, Truelove J, Tricot B, Wojtkiewicz G, Dutta P, Sager HB, et al: In vivo silencing of the transcription factor IRF5 reprograms the macrophage phenotype and improves infarct healing. J Am Coll Cardiol 63: 1556-1566, 2014.

13. Maier HJ, Schips TG, Wietelmann A, Krüger M, Brunner C, Sauter M, Klingel K, Böttger $\mathrm{T}$, Braun $\mathrm{T}$ and Wirth $\mathrm{T}$ : Cardiomyocyte-specific I $\kappa \mathrm{B}$ kinase $(\mathrm{IKK}) / \mathrm{NF}-\kappa \mathrm{B}$ activation induces reversible inflammatory cardiomyopathy and heart failure. Proc Natl Acad Sci USA 109: 11794-11799, 2012.

14. Smyth GK: Limma: Linear Models for Microarray Data. In: Bioinformatics and Computational Biology Solutions Using $\mathrm{R}$ and Bioconductor. Gentleman R, Carey VJ, Huber W, Irizarry RA and Dudoit S (eds). Springer, New York, NY, 2005.

15. Phipson B, Lee S, Majewski IJ, Alexander WS and Symth GK: Empirical Bayes in the presence of exceptional cases, with application to microarray data. ScienceOpen, Inc., Burlington, MA, 2016.

16. Ogata H, Goto S, Sato K, Fujibuchi W, Bono H and Kanehisa M: KEGG: Kyoto encyclopedia of genes and genomes. Nucleic Acids Res 27: 29-34, 1999.

17. Huang da W, Sherman BT and Lempicki RA: Systematic and integrative analysis of large gene lists using DAVID bioinformatics resources. Nat Protoc 4: 44-57, 2009.

18. Szklarczyk D, Franceschini A, Kuhn M, Simonovic M, Roth A, Minguez P, Doerks T, Stark M, Muller J, Bork P, et al: The STRING database in 2011: Functional interaction networks of proteins, globally integrated and scored. Nucleic Acids Res 39: D561-D568, 2011.
19. Shannon P, Markiel A, Ozier O, Baliga NS, Wang JT, Ramage D, Amin N, Schwikowski B and Ideker T: Cytoscape: A software environment for integrated models of biomolecular interaction networks. Genome Res 13: 2498-2504, 2003.

20. Bandettini WP, Kellman P, Mancini C, Booker OJ, Vasu S, Leung SW, Wilson JR, Shanbhag SM, Chen MY and Arai AE: MultiContrast delayed enhancement (MCODE) improves detection of subendocardial myocardial infarction by late gadolinium enhancement cardiovascular magnetic resonance: A clinical validation study. J Cardiovasc Magn Reson 14: 83, 2012.

21. Wang J, Duncan D, Shi Z and Zhang B: WEB-based GEne SeT AnaLysis Toolkit (WebGestalt): Update 2013. Nucleic Acids Res 41: W77-W83, 2013.

22. Livak KJ and Schmittgen TD: Analysis of relative gene expression data using real-time quantitative PCR and the 2(-Delta Delta C(T)) method. Method 25: 402-408, 2001.

23. Franics GS and Tang WH: Pathophysiology of congestive heart failure. Rev Cardiovasc Med 4 (Suppl 2): S14-S20, 2003.

24. Ziaeian B and Fonarow GC: Epidemiology and aetiology of heart failure. Nat Rev Cardiol 13: 368-378, 2016.

25. Marrouche NF, Brachmann J, Andresen D, Siebles J, Boersma L, Jordaens L, Merkely B, Pokushalov E, Sanders P, Proff J, et al: Catheter ablation for atrial fibrillation with heart failure. N Engl J Med 378: 417-427, 2018.

26. Velazquez EJ, Morrow DA, DeVore AD, Duffy CI, Ambrosy AP, McCague K, Rocha R, Braunwald E and PIONEER-HF Investigators: Angiotensin-neprilysin inhibition in acute decompensated heart failure. N Engl J Med 380: 539-548, 2019.

27. Caldwell MD, Hu SJ, Viswanathan S, Bradshaw H, Kelly ME and Straiker A: A GPR18-based signalling system regulates IOP in murine eye. Br J Pharmacol 169: 834-843, 2013.

28. Alexander SP: So what do we call GPR 18 now? Br J Pharmacol 165: 2411-2413, 2012.

29. Penumarti A and Abdel-Rahman AA: Role of central atypical cannabinoid receptor GPR18 in modulating cardiovascular function. FASEB J 26: (Suppl 1): 663.10, 2012.

30. Penumarti A and Abdel-Rahman AA: Abstract 17612: Central GPR18 mediates hypotension via enhanced PI3K/AKT-ERK1/2-nNOS signaling and inhibition of cAMP in the rostral ventrolateral medulla of conscious normotensive rats. Circulation 128: A17612-A17612, 2013.

31. Sheydina A, Volkova M, Jiang L, Juhasz O, Zhang J, Tae HJ, Perino MG, Wang M, Zhu Y, Lakatta EG and Boheler KR: Linkage of cardiac gene expression profiles and ETS2 with lifespan variability in rats. Aging Cell 11: 350-359, 2012.

32. Islas JF, Liu Y, Weng KC, Robertson MJ, Zhang S, Prejusa A, Harger J, Tikhomirova D, Chopra M, Iyer D, et al: Transcription factors ETS2 and MESP1 transdifferentiate human dermal fibroblasts into cardiac progenitors. Proc Natl Acad Sci USA 109: 13016-13021, 2012.

33. Schwartz RJ, Potaman VN and Francisco IJ: Ets2 and Mesp1 generate cardiac progenitors from fibroblasts. US Patent 9109232. Filed June 12, 2013; issued August 18, 2015.

34. Rowell J, Koitabashi N, Kass DA and Barth AS: Dynamic gene expression patterns in animal models of early and late heart failure reveal biphasic-bidirectional transcriptional activation of signaling pathways. Physiol Genomics 46: 779-787, 2014.

35. Czyzyk-Krzeska MF and Zhang X: MiR-155 at the heart of oncogenic pathways. Oncogene 33: 677-678, 2014.

36. Bao JL and Lin L: MiR-155 and miR-148a reduce cardiac injury by inhibiting NF- $\kappa \mathrm{B}$ pathway during acute viral myocarditis. Eur Rev Med Pharmacol Sci 18: 2349-2356, 2014.

37. Blanco RR, Austin H, Vest RN III, Valadri R, Li W, Lassegue B, Song Q, London B, Dudley SC, Bloom HL, et al: Angiotensin receptor type 1 single nucleotide polymorphism A1166C is associated with malignant arrhythmias and altered circulating miR-155 levels in patients with chronic heart failure. J Card Fail 18: 717-723, 2012.

38. Matsumoto S, Sakata Y, Nakatani D, Suna S, Mizuno H, Shimizu M, Usami M, Sasaki T, Sato H, Kawahara Y, et al: A subset of circulating microRNAs are predictive for cardiac death after discharge for acute myocardial infarction. Biochem Biophys Res Commun 427: 280-284, 2012.

(i) $($ ) This work is licensed under a Creative Commons Attribution-NonCommercial-NoDerivatives 4.0 International (CC BY-NC-ND 4.0) License. 